\title{
As maloclusões concedem aos indivíduos um perfil de suspeito a um crime?
}

\author{
Do maloclusions give individuals a profile of suspicious crime? \\ ¿Las maloclusiones le dan a las personas un perfil de crimen sospechoso?
}

Recebido: 02/08/2021 | Revisado: 10/08/2021 | Aceito: 06/10/2021 | Publicado: 12/10/2021

Nathalia de Lima Santos
ORCID: https://orcid.org/0000-0001-6829-3805
Eniversidade Estadual do Sudoeste da Bahia, Brasil
E-mail: tnlima@ hotmail.com
Camila Barreto Rangel dos Santos
ORCID: https://orcid.org/0000-0002-2633-6501
Universidade Estadual do Sudoeste da Bahia, Brasil
E-mail: milarangel16@gmail.com
Stefanni Olga Aguiar Sales Lima
ORCID: https://orcid.org/0000-0002-5583-5668
Universidade Estadual do Sudoeste da Bahia, Brasil
E-mail: stefanniolga@gmail.com
Fernanda Michel Tavares Canto
ORCID: https://orcid.org/0000-0002-7058-5724
Universidade Federal do Rio de Janeiro, Brasil
E-mail:nanda_michel@ hotmail.com
João Pedro Pedrosa Cruz
ORCID: https://orcid.org/0000-0003-3326-0469
Universidade Estadual do Sudoeste da Bahia, Brasil
E-mail: jpcruz@hotmail.com
Ana Carolina Dias Viana de Andrade
ORCID: https://orcid.org/0000-0001-6172-5652
Universidade Estadual do Sudoeste da Bahia, Brasil
E-mail: caroldvaa@yahoo.com.br
Raildo da Silva Coqueiro
ORCID: https://orcid.org/0000-0003-2278-1234
Universidade Estadual do Sudoeste da Bahia, Brasil
E-mail:matheuspithon@gmail.com
E-maiversidade Estadual do Sudoeste da Bahia, Brasil
Matheoueiro@uesb.edu.br
ORCID: Mttps://rcid.org/000-0002-8 Pithon

\section{Resumo}

O objetivo desse estudo foi avaliar a influência das maloclusões classe I (diastema e apinhamento) em conceder aos indivíduos, um perfil de caráter suspeito a um crime, por agentes de segurança pública. Foi realizado um estudo transversal com agentes de segurança de ambos os sexos $(n=100)$. Utilizou-se para o estudo imagens de retratos falados de um mesmo indivíduo com diferentes tipos de maloclusão: classe I com diastema e apinhamento e sorriso ideal. De posse dessas imagens criou-se um questionário dividido em duas partes. A primeira onde as imagens foram apresentadas todas juntas onde permitia a comparação entre elas; e a segunda onde cada imagem foi avaliada separadamente, seguida de perguntas e de uma escala visual analógica que apresentava uma barra com marcações que iam de 0 a 100, onde 0 representava o indivíduo não confiável, 50 confiável e 100 muito confiável. Através dos dados obtidos, seguiu-se a realização das análises estatísticas utilizando-se os testes qui-quadradro e de Friedman. O nível de significância adotado foi de $5 \%(\alpha=0,05)$. O indivíduo com sorriso ideal foi associado pelos agentes de segurança como o mais propenso a cometer crimes, quando comparado aos indivíduos com diastema e apinhamento $(\mathrm{p}<0,001)$. $\mathrm{O}$ indivíduo com sorriso ideal foi associado pelos agentes de segurança como os mais propensos a cometer crimes, devido a exibir traços mais maduros, quando comparados a indivíduos com maloclusões classe I, que atribui-lhe característica morfológica infantil.

Palavras chave: Percepção social; Odontologia legal; Comportamento criminoso; Maloclusão.

\section{Abstract}

To evaluate the influence of class I malocclusions (diastema and crowding) in granting to public security agents. A cross-sectional study was carried out with security officers of both sexes $(n=100)$. It was used to study images of sketch of the same individual with class I malocclusion with diastema and crowding and ideal smile. With these images in hand, a questionnaire was created, divided into two parts, the first where the images were put together to 
allow the comparison between them and the second where each image was evaluated following the questions and a visual analogue scale that presented a bar with markings ranging from 0 to 100 where 0 represented the untrustworthy individual, 50 trusted and 100 very trustworthy. With the obtained data, statistical analyzes were carried out using the chi-square and Friedman tests. The significance level adopted was 5\% $(\alpha=0.05)$. The individual with the ideal smile was associated by security agents as more prone to common crimes, when compared to individuals with diastema and crowding ( $\mathrm{p}<0.001)$. The individual with ideal smile was associated by security agents as the most likely to commit crimes, due to exhibiting more mature traits, when compared to disability with class I malocclusions, which gives them the child's morphological characteristic.

Keywords: Social perception; Forensic dentistry; Criminal behavior; Maloclusion.

\section{Resumen}

Evaluar la influencia de las maloclusiones clase I (diastema y hacinamiento) en dar a los individuos un perfil de carácter sospechoso por un delito para los agentes de seguridad pública. Se realizó un estudio transversal con agentes de seguridad de ambos sexos $(n=100)$. Para el estudio se utilizaron imágenes de retratos hablados del mismo individuo con maloclusión clase I con diastema y apiñamiento y sonrisa ideal. Con estas imágenes en la mano, se elaboró un cuestionario, dividido en dos partes, la primera donde se presentaron las imágenes todas juntas permitiendo una comparación entre ellas y la segunda donde cada imagen se evaluó por separado seguido de preguntas y una escala visual analógica que presentó una barra con marcas que van de 0 a 100 , donde 0 representa al individuo no confiable, 50 confiable y 100 muy confiable. Con los datos obtenidos se realizaron análisis estadísticos mediante las pruebas de chi-cuadrado y Friedman. El nivel de significancia adoptado fue del 5\% $(\alpha=0.05)$. Los agentes de seguridad asociaron al individuo con sonrisa ideal como el más propenso a cometer delitos, en comparación con los individuos con diastema y hacinamiento $(\mathrm{p}<0,001)$. El individuo con sonrisa ideal fue asociado por los agentes de seguridad como el más propenso a cometer delitos, por exhibir rasgos más maduros, en comparación con los individuos con maloclusiones clase I, lo que les confiere una característica morfológica infantil.

Palabras clave: Percepción social; Odontología forense; Conducta criminal; Maloclusión.

\section{Introdução}

A percepção facial é uma habilidade extremamente bem desenvolvida entre os seres humanos, e é um dado essencial para as interações sociais. Reunimos uma riqueza de informações socialmente relevantes, tais como a emoção e familiaridade, a partir da face. As pesquisas comportamentais tem demonstrado que os seres humanos formam rapidamente uma série de atribuições sociais, a julgar a confiabilidade e a atratividade (Stirrat \& Perrett, 2010).

A confiabilidade do alvo é relevante para o julgamento judicial (Wilson \& Rule, 2015), visto que umas das decisões mais críticas que um agente de segurança pode tomar é atirar ou não atirar em um suspeito criminoso(Kenrick, Griskevicius, Neuberg, \& Schaller, 2010; Neuberg, Kenrick, \& Schaller, 2011), e que a morte de uma pessoa inocente, é motivo suficiente para justificar a investigação sobre os fatores que determinam as decisões desse profissional (Miller, Zielaskowski, \& Plant, 2012).

Pesquisadores tem utilizado o termo criminalidade também para indicar a influência que a "a aparência de uma pessoa desencadeia sobre estereótipos criminosos" e compreender a natureza exata deste processo permanece um desafio, porque a face, como um transmissor de múltiplas e complexas informações sociais, compreende, um espaço de alta dimensão e informação dinâmica (Jack \& Schyns, 2015; Otto H. Maclin, 2004).

Embora as implicações estéticas da maloclusão sejam geralmente consideradas como sendo importantes (Shaw, Rees, Dawe, \& Charles, 1985), nenhuma pesquisa foi direcionada a essa característica na criminologia atual. Sendo assim, o objetivo do presente estudo foi avaliar se as maloclusões dentárias concedem aos indivíduos um perfil de caráter suspeito para agentes de segurança pública. Busca-se confirma com esse estudo a hipótese que os indivíduos com maloclusão apresentam um caráter suspeito maior para cometer crime.

\section{Metodologia}

Trata-se de um estudo transversal realizado com agentes de segurança de ambos os sexos com faixa etária entre 25-60 anos de idade no município de Jequié, Bahia, Brasil, os quais se propuseram a avaliar se os padrões faciais são características 
que tornam um indivíduo suspeito a ter cometido um crime. Excluiu-se da amostra agentes de segurança que apresentasse distúrbios oftalmológicos que atrapalhasse a avaliação das imagens.

Inicialmente realizou-se o cálculo do tamanho da amostra utilizando o nQueryAdviser (Versão estatísticas 6,01 Solution, York, Irlanda). Com base em uma $(\alpha=0,05)$ o nível de significância de $5 \%$, o tamanho da amostra foi calculado para alcançar o potencial de $80 \%$. O cálculo do tamanho da amostra mostrou que seria necessário um número de 100 agentes de segurança.

Previamente ao início da coleta de dados, o projeto foi enviado para aprovação ao Comité de ética da Universidade Estadual Sudoeste da Bahia e recebeu um relatório favorável (número 405.925). Todas os participantes que responderam aos questionários, assinaram o termo de consentimento livre e esclarecido.

Para realização desse estudo utilizou-se imagem de retrato falado de um mesmo indivíduo com diferentes maloclusões (diastema, apinhamento e sorriso ideal). De posse dessas criou-se um questionário. O questionário era composto por 22 perguntas fechadas associadas as imagens onde os participantes do estudo tiveram o tempo de um minuto para visualizar cada imagem, responder as perguntas propostas e preencher a escala, sem direito de retornar à imagem anterior.

Previamente à análise das imagens, foram feitas algumas perguntas aos avaliadores em relação ao sexo, idade, patente e tempo na polícia. Na primeira etapa do questionário as três imagens eram apresentadas todas juntas seguidas dos seguintes questionamentos (1) Qual desses indivíduos você acha que poderia ser o autor de algum crime? (2) Qual desses indivíduos passa mais insegurança/desconfiança? Na sequência do questionário as mesmas imagens foram reapresentadas em posições alteradas seguidas das mesmas perguntas. Essa etapa inicial do questionário teve por objetivo avaliar a confiabilidade das respostas dadas pelos avaliadores.

Na sequência, cada imagem foi apresentada de modo individualizada seguida pelos seguintes questionamentos: (1) Durante uma abordagem, você consideraria este indivíduo como suspeito de cometer um crime? (2) Este indivíduo aparenta ser uma pessoa honesta? (3) Caso esse indivíduo tivesse cometido um crime, qual ou quais crimes você atribuiria a ele? (4) Você acredita que essa pessoa reagiria com violência a uma abordagem policial? (5) Numa situação em que você e esse indivíduo estivesse desarmado, você ficaria com receio de enfrenta-lo em uma luta corporal? Após esses questionamentos os avaliadores foram convidados a dar uma nota relacionada a confiança passada pela imagem do retrato falado utilizando pra isso uma Escala Visual Analógica (EVA) 0-100mm. Esta escala apresenta uma barra com marcações que vão de 0 a 100 onde 0 representava o indivíduo não confiável, 50 confiável e 100 muito confiável. O valor numérico correspondente foi anotado para ser tabulado e avaliado estatisticamente. Para responder a todas as questões os avaliadores tinham as alternativas: não, sim e não sei, exceto pela questão 3 que possuía as opções de crimes mais comuns, permitindo assim que os avaliadores assinalassem mais de uma alternativa.

Os questionários foram apresentados em um notebook (Toshiba, Tokyo, Japan) com tela de Light Emitting Diode (LED) 15 polegadas que ficou a uma distância de $30 \mathrm{~cm}$ do avaliador.

\subsection{Análise Estatística}

As frequências das respostas dadas pelos participantes foram comparadas por meio do teste qui-quadradro. Os escores das notas atribuídas as imagens foram comparados por meio do teste Friedman. O nível de significância adotado foi de 5\% $(\alpha=0,05)$. Os dados foram tabulados e analisados no IBM SPSS Statistics for Windows (IBM SPSS. 21.0, 2012, Armonk, NY: IBM Corp.). 


\section{Resultados}

A idade dos participantes variou de 26 a 65 anos, com a média sendo de 38,2 $\pm 6,2$ anos. O tempo médio de profissão foi de 13,9 $\pm 5,5$ anos ( 3 - 30 anos). Na Tabela 1 são mostradas as demais características da amostra.

Tabela 1. Dados sociodemográficos dos participantes do estudo.

\begin{tabular}{lcc}
\hline Variáveis & $\mathrm{n}$ & $\%$ \\
\hline Sexo & & \\
Masculino & 80 & 80,0 \\
Feminino & 20 & 20,0 \\
Patente & & \\
Soldado & 72 & 72,0 \\
Sargento & 13 & 13,0 \\
Tenente & 9 & 9,0 \\
Capitão & 6 & 6,0 \\
& & \\
\hline
\end{tabular}

Fonte: Autores.

Na Figura 1 é apresentada a percepção dos participantes da pesquisa a respeito da possibilidade de cometer crime e insegurança/desconfiança correspondente as imagens apresentadas. De acordo com a percepção dos policiais entrevistados, o indivíduo representado na imagem $\mathrm{C}$ (sorriso ideal) foi associado a maior possibilidade de cometer crime, quando comparado aos indivíduos representados na imagem A (portador de diastema) e B (portador de apinhamento). Não foi observada diferença significativa com relação à insegurança/desconfiança passada pelos indivíduos representados nas três imagens.

Figura 1: Imagem A (portador de diastema); Imagem B (portador de apinhamento); Imagem C( sorriso ideal).

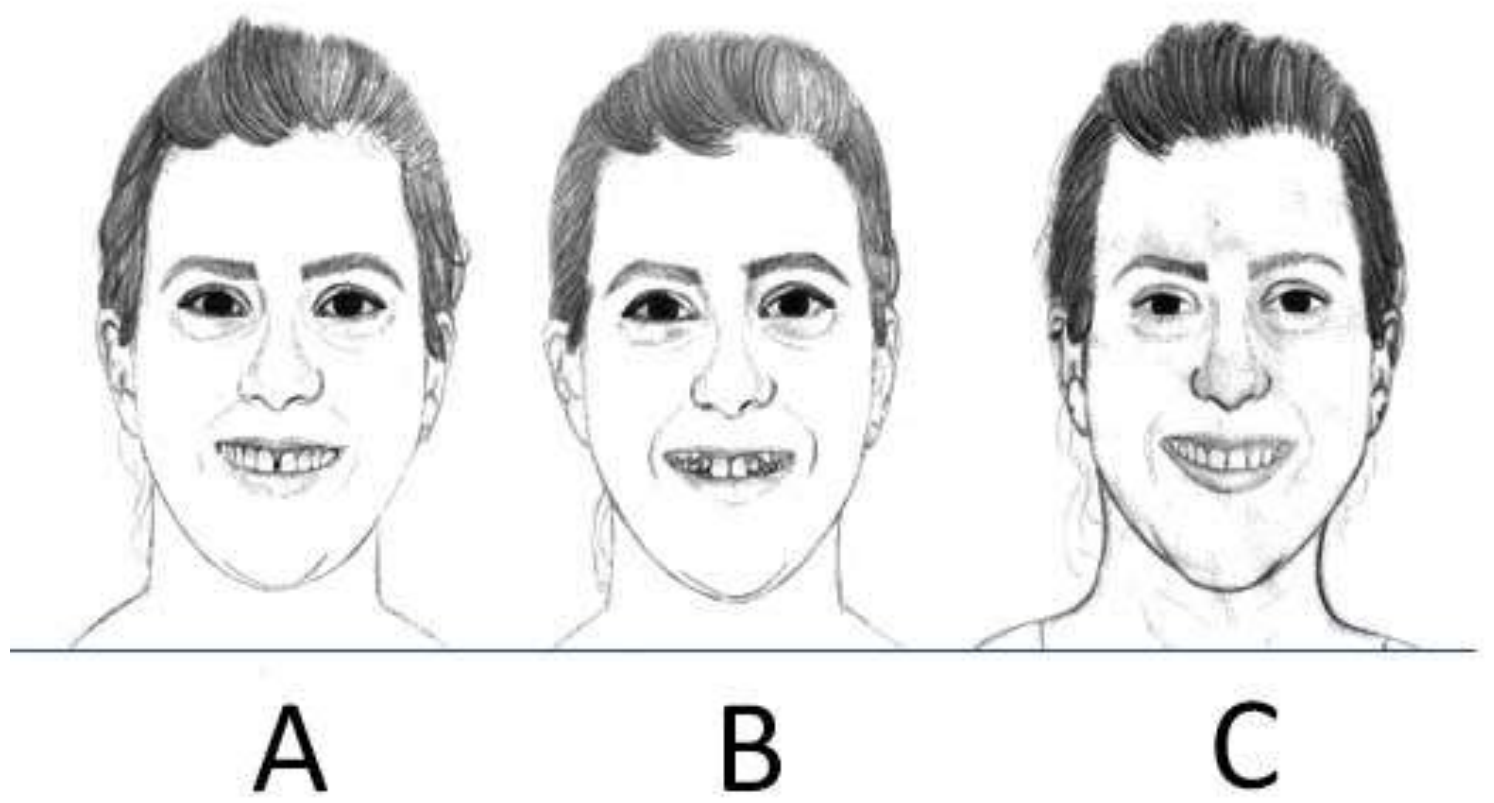

Imagem A (portador de diastema); Imagem B (portador de apinhamento); Imagem C (sorriso ideal). Fonte: Stefanni Olga Aguiar Sales Lima. 
Na Tabela 2 é apresentada a percepção dos participantes da pesquisa a respeito das questões realizadas sobre os perfis dos indivíduos apresentados nas imagens. Foram observadas diferenças significativas entre as imagens apenas para as questões sobre atribuição do crime de roubo simples e atribuição do crime de tráfico de entorpecentes. De acordo com a percepção dos policiais entrevistados, o indivíduo representado na imagem B (portador de apinhamento) foi associado a maior possibilidade de cometer crime de roubo simples, enquanto que o indivíduo representado na imagem $\mathrm{C}$ (sorriso ideal) foi associado a maior possibilidade de cometer crime de tráfico de entorpecentes.

Tabela 2. Respostas dos participantes do estudo, de acordo com as questões da pesquisa sobre os perfis dos indivíduos apresentados nas imagens.

\begin{tabular}{|c|c|c|c|c|}
\hline \multirow{2}{*}{ Pergunta } & \multicolumn{3}{|c|}{ Imagem } & \multirow{2}{*}{ p-valor } \\
\hline & A & $\mathrm{B}$ & $\mathrm{C}$ & \\
\hline \multicolumn{5}{|c|}{ Suspeito de crime } \\
\hline Sim & $43(43 \%)$ & $39(39 \%)$ & $40(40 \%)$ & \multirow{3}{*}{0,645} \\
\hline Não & $17(17 \%)$ & $15(15 \%)$ & $22(22 \%)$ & \\
\hline Não sei & $40(40 \%)$ & $46(46 \%)$ & $38(38 \%)$ & \\
\hline \multicolumn{5}{|c|}{ Aparenta ser pessoa honesta } \\
\hline Sim & $20(20 \%)$ & $23(23 \%)$ & $26(26 \%)$ & \multirow{3}{*}{0,309} \\
\hline Não & $22(22 \%)$ & $19(19 \%)$ & $11(11 \%)$ & \\
\hline Não sei & $58(58 \%)$ & $58(58 \%)$ & $63(63 \%)$ & \\
\hline \multicolumn{5}{|c|}{ Crime atribuído: roubo simples } \\
\hline Sim & $22(22 \%)$ & $35(35 \%)$ & $20(20 \%)$ & \multirow{2}{*}{0,031} \\
\hline Não & $78(78 \%)$ & $65(65 \%)$ & $80(80 \%)$ & \\
\hline \multicolumn{5}{|c|}{ Crime atribuído: roubo qualificado } \\
\hline Sim & $23(23 \%)$ & $14(14 \%)$ & $14(14 \%)$ & \multirow{2}{*}{0,148} \\
\hline Não & $77(77 \%)$ & $86(86 \%)$ & $86(86 \%)$ & \\
\hline \multicolumn{5}{|c|}{ Crime atribuído: furto simples } \\
\hline Sim & $44(44 \%)$ & $41(41 \%)$ & $31(31 \%)$ & \multirow{2}{*}{0,142} \\
\hline Não & $56(56 \%)$ & $59(59 \%)$ & $69(69 \%)$ & \\
\hline \multicolumn{5}{|c|}{ Crime atribuído: furto qualificado } \\
\hline Sim & $18(18 \%)$ & $10(10 \%)$ & $15(15 \%)$ & \multirow{2}{*}{0,264} \\
\hline Não & $82(82 \%)$ & $90(90 \%)$ & $85(85 \%)$ & \\
\hline \multicolumn{5}{|c|}{ Crime atribuído: homicídio simples } \\
\hline Sim & $13(13 \%)$ & $17(17 \%)$ & $12(12 \%)$ & \multirow{2}{*}{0,559} \\
\hline Não & $87(87 \%)$ & $83(83 \%)$ & $88(88 \%)$ & \\
\hline \multicolumn{5}{|c|}{ Crime atribuído: homicídio qualificado } \\
\hline Sim & $15(15 \%)$ & $11(11 \%)$ & $22(22 \%)$ & \multirow{2}{*}{0,100} \\
\hline Não & $85(85 \%)$ & $89(89 \%)$ & $78(78 \%)$ & \\
\hline \multicolumn{5}{|c|}{ Crime atribuído: porte ilegal de arma } \\
\hline Sim & $30(30 \%)$ & $31(31 \%)$ & $25(25 \%)$ & \multirow{2}{*}{0,603} \\
\hline Não & $70(70 \%)$ & $69(69 \%)$ & $75(75 \%)$ & \\
\hline \multicolumn{5}{|c|}{ Crime atribuído: tráfico de entorpecentes } \\
\hline Sim & $34(34 \%)$ & $29(29 \%)$ & $57(57 \%)$ & \multirow{2}{*}{$<0,001$} \\
\hline Não & $66(66 \%)$ & $71(71 \%)$ & $43(43 \%)$ & \\
\hline \multicolumn{5}{|c|}{ Crime atribuído: latrocínio } \\
\hline Sim & $11(11 \%)$ & $18(18 \%)$ & $11(11 \%)$ & \\
\hline Não & $89(89 \%)$ & $82(82 \%)$ & $89(89 \%)$ & 0,243 \\
\hline Reação vi & & & & \\
\hline $\operatorname{Sim}^{3}$ & $26(26 \%)$ & $25(25 \%)$ & $26(26 \%)$ & \\
\hline Não & $28(28 \%)$ & $28(28 \%)$ & $25(25 \%)$ & 0,987 \\
\hline Não sei & $46(46 \%)$ & $47(47 \%)$ & $49(49 \%)$ & \\
\hline Receio de & & & & \\
\hline Sim & $24(24 \%)$ & $22(22 \%)$ & $22(22 \%)$ & \\
\hline Não & $51(51 \%)$ & $56(56 \%)$ & $59(59 \%)$ & 0,822 \\
\hline Não sei & $25(25 \%)$ & $22(22 \%)$ & $19(19 \%)$ & \\
\hline
\end{tabular}

Fonte: Autores. 
A comparação das notas entre as imagens com relação a confiança passada é mostrada na Tabela 2. Não foi observada diferença significava entre as imagens, indicando que a presença de apinhamento e diastema não influenciou a percepção dos policiais sobre a confiança passada pelos indivíduos representados nas imagens.

\section{Discussão}

A aparência física tem grande influência sobre a integração social de uma pessoa, contribuindo para sua qualidade de vida, auto-estima e atratividade física(Palomares, Celeste, Oliveira, \& Miguel, 2012). Um corpo crescente da literatura científica, tem sugerido que a aparência física de um indivíduo afeta sistematicamente atitudes e ações sociais(Eli, Bar-Tal, \& Kostovetzki, 2001). Sendo a face o componente do corpo humano mais aparente e consequentemente mais julgada, os rostos influenciam significativamente nas classificações de inteligência, sociabilidade, moralidade, atratividade e confiabilidade (Alicke MD, 1986; Naini, Cobourne, McDonald, \& Donaldson, 2008; Palomares et al., 2012)(Flowe, 2012; Neth \& Martinez, 2009; Oosterhof \& Todorov, 2008; Said, Sebe, \& Todorov, 2009).

Dessa forma, as pessoas inferem confiabilidade a determinados rostos de forma rápida e com alto consenso(Willis \& Todorov, 2006), sendo isso observado mesmo quando informações comportamentais estão disponíveis(Rudoy \& Paller, 2009; Todorov \& Olson, 2008). Traços morfologicamente maduros são julgados como fisicamente mais fortes, mais dominantes, e menos honestos, em contraposição com rostos que trazem traços morfologicamente infantis (Todorov, Said, Engell, \& Oosterhof, 2008).

Para agentes de segurança pública, visto que, embora as implicações estéticas das maloclusões sejam geralmente consideradas como sendo importantes(Shaw et al., 1985), poucas pesquisas foram direcionadas a estudar a influência dessas características na criminologia atual. Para a realização do estudo optou-se pela exposição de retrato falado de um mesmo indivíduo, com alterações dentárias, como visto nos resultados, pois esta é a ferramenta mais comumente utilizada por agentes de segurança quando da captura de um foragido.

Ao avaliar os retratos falados (Figura 1) que apresentavam diastema, apinhamento e sorriso ideal, o indivíduo com sorriso sem alterações de maloclusão classe I foi associado pelos agentes de segurança como mais propenso a cometer crimes. $\mathrm{Na}$ Tabela. 2 foi possível perceber que a percepção dos participantes da pesquisa a respeito da atribuição do crime de roubo simples e de tráfico de entorpecentes sobre os perfis dos indivíduos teve grande significância, visto que, o indivíduo portador de apinhamento foi associado a maior possibilidade de cometer crime de roubo simples, enquanto que o indivíduo com sorriso ideal foi associado a maior possibilidade de cometer crime de tráfico de entorpecentes. Tais resultados, estão associados a grande incidência de maloclusões classe I em crianças (Martins Mda \& Lima, 2009), e o fato de que traços infantis são visto como mais inofensivos, menos agressivos e denotam fragilidade (Hahn \& Perrett, 2014) em contraposição com traços maduros, que são julgados como fisicamente mais fortes, mais dominantes, e menos honestos (Todorov et al., 2008), embora quando analisamos interações sociais, alguns estudos trazem maloclusões dentárias como ponto negativo (Henson et al., 2011; Pithon, Nascimento, Barbosa, \& Coqueiro Rda, 2014).

Podemos levar em consideração o fato de que características físicas de criminosos são geralmente estereotipadas (Flowe, Klatt, \& Colloff, 2014) e uma identificação criminal através de uma testemunha ocular "deve ser baseado na memória". No entanto, os estereótipos criminais podem influenciar os resultados de uma identificação, na qual as testemunhas selecionam as pessoas de acordo a suas concepções pessoais de traços criminosos, sendo a avaliação da atratividade facial subjetiva (Hills, Lewis, \& Honey, 2008; Peck \& Peck, 1970)

Os resultados desse estudo se juntam àqueles de outras pesquisas sobre o papel da informação facial na sentença penal (Blair, Judd, \& Chapleau, 2004; Eberhardt, Davies, Purdie-Vaughns, \& Johnson, 2006), atribuindo um dado alarmante acerca de maloclusões dentárias em julgamentos de alto impacto (sentenças criminais), com o objetivo de diminuir os vieses(Wilson 
\& Rule, 2015) investigativos, e provocar nos agentes de segurança um olhar criterioso e atencioso ao julgarem relatos de testemunhas oculares, embora, mais pesquisas sejam necessárias para determinar o processo através do qual a aparência influencia os resultados criminais e solidificar a estrutura causal dessa relação.

\section{Conclusão}

Diante dos resultados obtidos, é possível concluir que o indivíduo com sorriso ideal foi associado pelos agentes de segurança como os mais propensos a cometer crimes, devido a exibir traços mais maduros, quando comparados a indivíduos com maloclusões classe I, que atribui-lhe característica morfológica infantil.

\section{Referências}

Alicke, M. D, \& Klotz, M. L. (1986). Judgments of physical attractiveness: the role of faces and bodies. Pers Soc Psychol Bd, 12, 381-389.

Blair, I. V., Judd, C. M., \& Chapleau, K. M. (2004). The influence of Afrocentric facial features in criminal sentencing. Psychol Sci, 15(10), 674-679.

Eberhardt, J. L., Davies, P. G., Purdie-Vaughns, V. J., \& Johnson, S. L. (2006). Looking deathworthy: perceived stereotypicality of Black defendants predicts capital-sentencing outcomes. Psychol Sci, 17(5), 383-386.

Eli, I., Bar-Tal, Y., \& Kostovetzki, I. (2001). At first glance: social meanings of dental appearance. J Public Health Dent, 61(3), $150-154$.

Flowe, H. D. (2012). Do characteristics of faces that convey trustworthiness and dominance underlie perceptions of criminality? PLoS One, 7(6), e37253.

Flowe, H. D., Klatt, T., \& Colloff, M. F. (2014). Selecting fillers on emotional appearance improves lineup identification accuracy. Law Hum Behav, 38(6), 509-519.

Hahn, A. C., \& Perrett, D. I. (2014). Neural and behavioral responses to attractiveness in adult and infant faces. Neurosci Biobehav Rev, 46 Pt 4, $591-603$.

Henson, S. T., Lindauer, S. J., Gardner, W. G., Shroff, B., Tufekci, E., \& Best, A. M. (2011). Influence of dental esthetics on social perceptions of adolescents judged by peers. Am J Orthod Dentofacial Orthop, 140(3), 389-395.

Hills, P. J., Lewis, M. B., \& Honey, R. C. (2008). Stereotype priming in face recognition: interactions between semantic and visual information in face encoding. Cognition, 108(1), 185-200.

Jack, R. E., \& Schyns, P. G. (2015). The Human Face as a Dynamic Tool for Social Communication. Curr Biol, 25(14), R621-634.

Kenrick, D. T., Griskevicius, V., Neuberg, S. L., \& Schaller, M. (2010). Renovating the Pyramid of Needs: Contemporary Extensions Built Upon Ancient Foundations. Perspect Psychol Sci, 5(3), 292-314.

Martins Mda, G., \& Lima, K. C. (2009). Prevalence of malocclusions in 10- to 12-year-old schoolchildren in Ceara, Brazil. Oral Health Prev Dent, 7(3), 217223.

Miller, S. L., Zielaskowski, K., \& Plant, E. A. (2012). The basis of shooter biases: beyond cultural stereotypes. Pers Soc Psychol Bull, 38(10), $1358-1366$.

Naini, F. B., Cobourne, M. T., McDonald, F., \& Donaldson, A. N. (2008). The influence of craniofacial to standing height proportion on perceived attractiveness. Int J Oral Maxillofac Surg, 37(10), 877-885.

Neth, D., \& Martinez, A. M. (2009). Emotion perception in emotionless face images suggests a norm-based representation. $J$ Vis, 9(1), 5 1-11.

Neuberg, S. L., Kenrick, D. T., \& Schaller, M. (2011). Human threat management systems: self-protection and disease avoidance. Neurosci Biobehav Rev, 35(4), 1042-1051.

Oosterhof, N. N., \& Todorov, A. (2008). The functional basis of face evaluation. Proc Natl Acad Sci U S A, 105(32), 11087-11092

Otto H. Maclin, M. K. M. (2004). The Effect of Criminality on Face Attractiveness, Typicality, Memorability and Recognition. North American Journal of Psychology, 6(1), 145-154.

Palomares, N. B., Celeste, R. K., Oliveira, B. H., \& Miguel, J. A. (2012). How does orthodontic treatment affect young adults' oral health-related quality of life? Am J Orthod Dentofacial Orthop, 141(6), 751-758.

Peck, H., \& Peck, S. (1970). A concept of facial esthetics. Angle Orthodontist, 40(4), 284-318.

Pithon, M. M., Nascimento, C. C., Barbosa, G. C., \& Coqueiro Rda, S. (2014). Do dental esthetics have any influence on finding a job? Am J Orthod Dentofacial Orthop, 146(4), 423-429.

Rudoy, J. D., \& Paller, K. A. (2009). Who can you trust? Behavioral and neural differences between perceptual and memory-based influences. Front Hum Neurosci, 3, 16.

Said, C. P., Sebe, N., \& Todorov, A. (2009). Structural resemblance to emotional expressions predicts evaluation of emotionally neutral faces. Emotion, 9(2), 260-264. 
Research, Society and Development, v. 10, n. 12, e197101318984, 2021

(CC BY 4.0) | ISSN 2525-3409 | DOI: http://dx.doi.org/10.33448/rsd-v10i13.18984

Shaw, W. C., Rees, G., Dawe, M., \& Charles, C. R. (1985). The influence of dentofacial appearance on the social attractiveness of young adults. Am J Orthod, $87(1), 21-26$.

Stirrat, M., \& Perrett, D. I. (2010). Valid facial cues to cooperation and trust: male facial width and trustworthiness. Psychol Sci, $21(3), 349-354$.

Todorov, A., \& Olson, I. R. (2008). Robust learning of affective trait associations with faces when the hippocampus is damaged, but not when the amygdala and temporal pole are damaged. Soc Cogn Affect Neurosci, 3(3), 195-203.

Todorov, A., Said, C. P., Engell, A. D., \& Oosterhof, N. N. (2008). Understanding evaluation of faces on social dimensions. Trends Cogn Sci, 12(12), 455460 .

Willis, J., \& Todorov, A. (2006). First impressions: making up your mind after a 100-ms exposure to a face. Psychol Sci, 17(7), 592-598.

Wilson, J. P., \& Rule, N. O. (2015). Facial Trustworthiness Predicts Extreme Criminal-Sentencing Outcomes. Psychol Sci, 26(8), $1325-1331$. 